Portland State University

PDXScholar

\title{
Data From: Diversity of ACRL Publications, Editorial Board Demographics: A report from ACRL's Publications Coordinating Committee
}

Association of College \& Research Libraries Publications Coordinating Committee

Follow this and additional works at: https://pdxscholar.library.pdx.edu/ulib_fac

Part of the Scholarly Communication Commons, and the Scholarly Publishing Commons Let us know how access to this document benefits you.

\section{Citation Details}

Ford, E., Kaspar, W. and Seiden, P. (2017). Data From: Diversity of ACRL Publications, Editorial Board Demographics: A report from ACRL's Publications Coordinating Committee. https://doi.org/10.15760/ libdata.1

This Dataset is brought to you for free and open access. It has been accepted for inclusion in Library Faculty Publications and Presentations by an authorized administrator of PDXScholar. Please contact us if we can make this document more accessible: pdxscholar@pdx.edu. 


\section{Default Report}

\section{Ed Boards Demographic Survey}

February 8th 2017, 1:21 pm PST

\section{Q1 - Please indicate on which Editorial Board you serve}

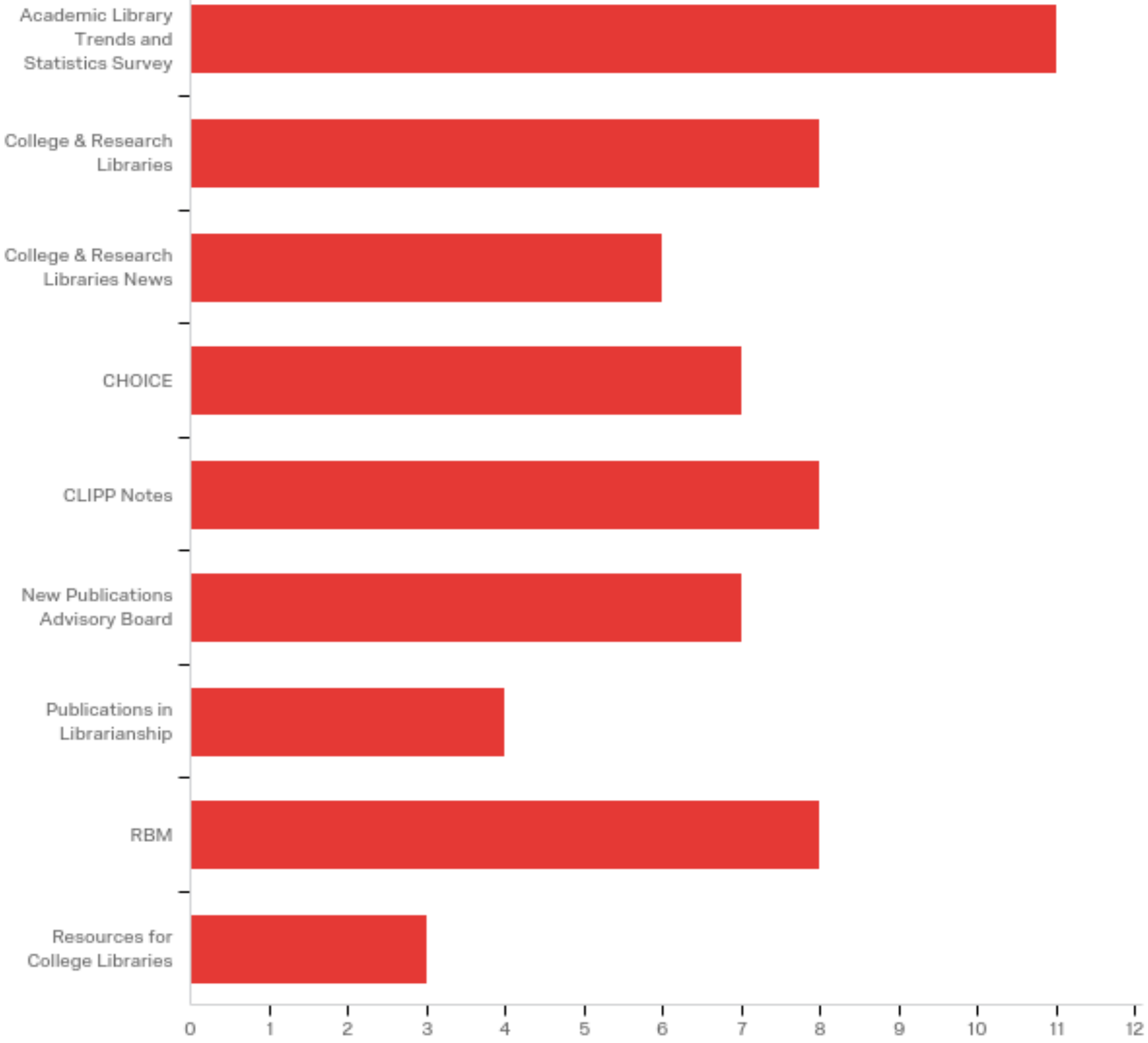

\begin{tabular}{r|r|r|r|}
\hline$\#$ & Answer & $\%$ & Count \\
\hline 1 & Academic Library Trends and Statistics Survey & $17.74 \%$ & 11 \\
\hline 2 & College \& Research Libraries & $12.90 \%$ & 8 \\
\hline
\end{tabular}




\begin{tabular}{|r|r|r|r|}
\hline 3 & College \& Research Libraries News & $9.68 \%$ & 6 \\
\hline 4 & CHOICE & $11.29 \%$ & 7 \\
\hline 5 & CLIPP Notes & $12.90 \%$ & 8 \\
\hline 6 & New Publications Advisory Board & $11.29 \%$ & 7 \\
\hline 9 & Publications in Librarianship & $6.45 \%$ & 4 \\
\hline 7 & RBM & $12.90 \%$ & 8 \\
\hline 8 & Resources for College Libraries & $4.84 \%$ & 3 \\
\hline & Total & $100 \%$ & 62 \\
\hline
\end{tabular}




\section{Q2 - Please choose your age range}

30 or under years old $31-40$ years old (2)

$$
-
$$

41-50 years old
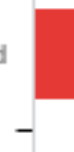

51-60 years old

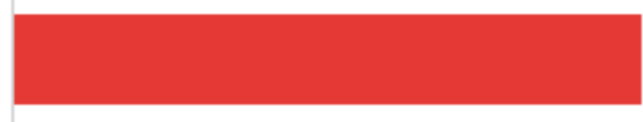

61 or over years old
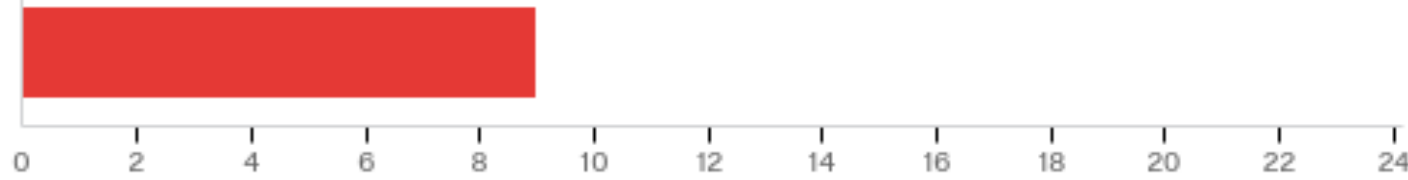

\begin{tabular}{|r|r|r|r|}
\hline$\#$ & Answer & $\%$ & Count \\
\hline 1 & 30 or under years old & $1.64 \%$ & 1 \\
\hline 2 & $31-40$ years old & $36.07 \%$ & 22 \\
\hline 3 & $41-50$ years old & $29.51 \%$ & 18 \\
\hline 4 & $51-60$ years old & $18.03 \%$ & 11 \\
\hline 5 & 61 or over years old & $14.75 \%$ & 9 \\
\hline & Total & $100 \%$ & 61 \\
\hline
\end{tabular}




\section{Q3 - Please select your gender}

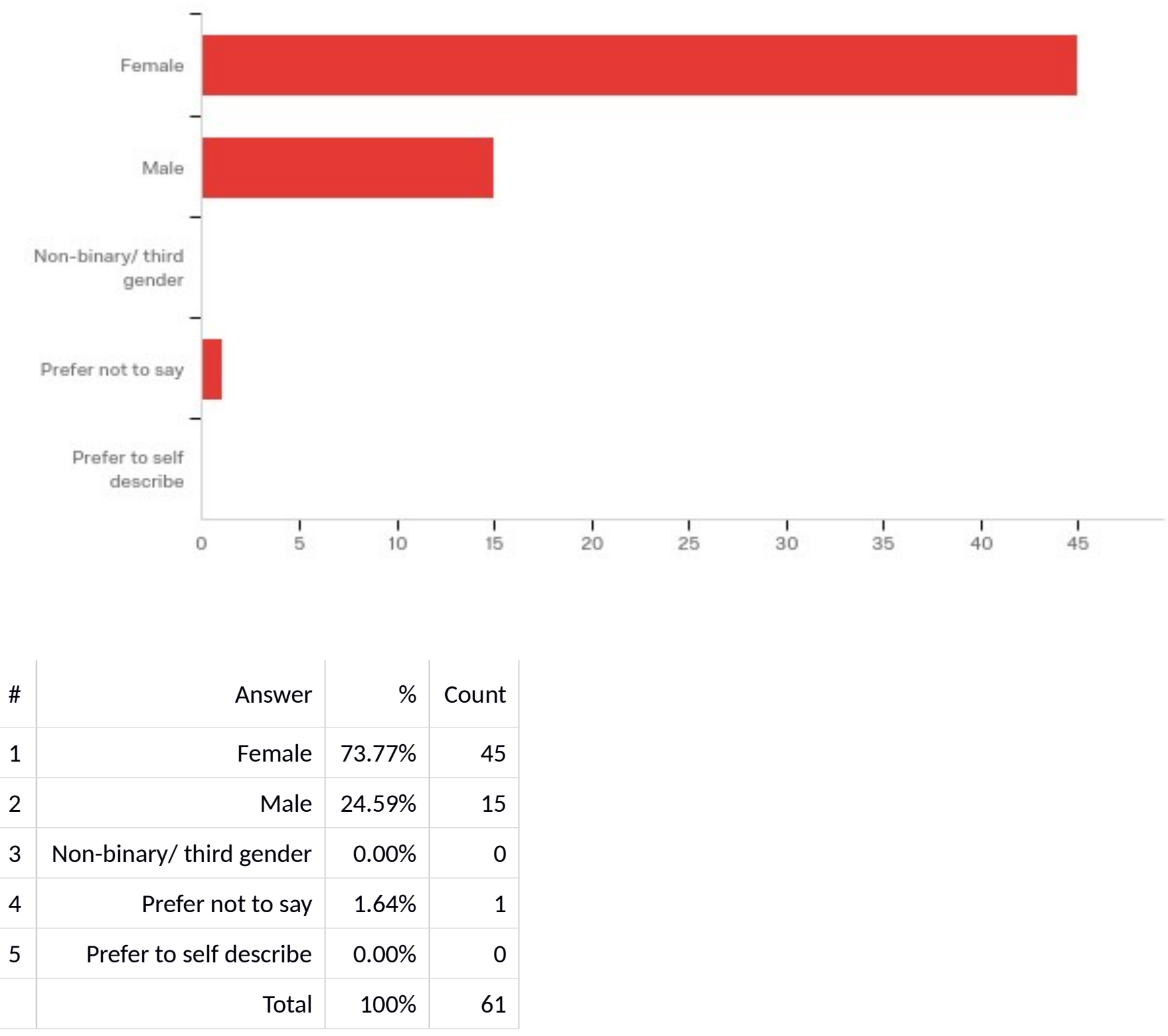

Prefer to self describe

Prefer to self describe 


\section{Q4 - Please select all appropriate options for your race/ethnicity}

American Indian or Alaska Native

Asian

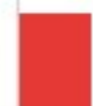

Black or African American .

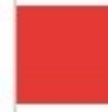
$-$

\section{Hispanic or Latino}

Native Hawaiian or Pacific Islander

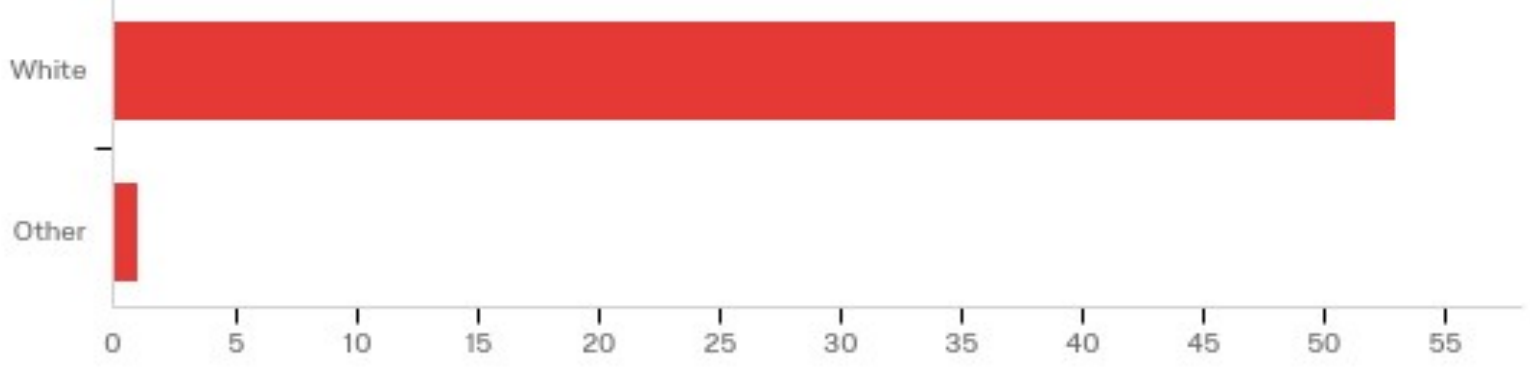

,

\begin{tabular}{r|r|r|r}
$\#$ & Answer & $\%$ & Count \\
\hline 1 & American Indian or Alaska Native & $0.00 \%$ & 0 \\
\hline 2 & Asian & $5.00 \%$ & 3 \\
\hline 3 & Black or African American & $6.67 \%$ & 4 \\
\hline 4 & Hispanic or Latino & $0.00 \%$ & 0 \\
\hline 5 & Native Hawaiian or Pacific Islander & $0.00 \%$ & 0 \\
\hline 6 & White & $88.33 \%$ & 53 \\
\hline 7 & Other & $1.67 \%$ & 1 \\
\hline & Total & $100 \%$ & 60 \\
\hline
\end{tabular}


Q5 - Please select the option that best describes the geographic location of your main institutional affiliation

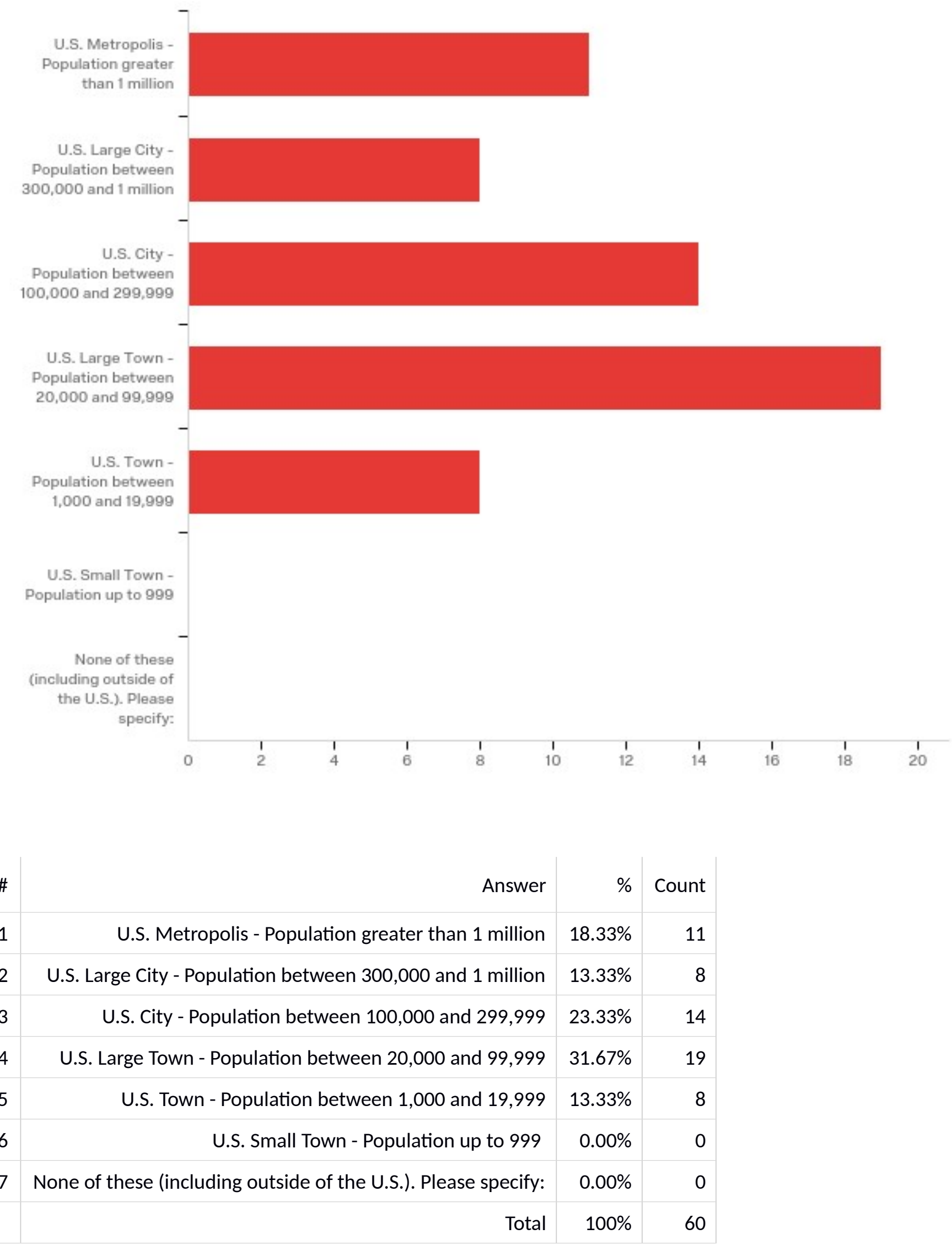


None of these (including outside of the U.S.). Please specify:

None of these (including outside of the U.S.). Please specify: 


\section{Q6 - Please choose your main professional affiliation}

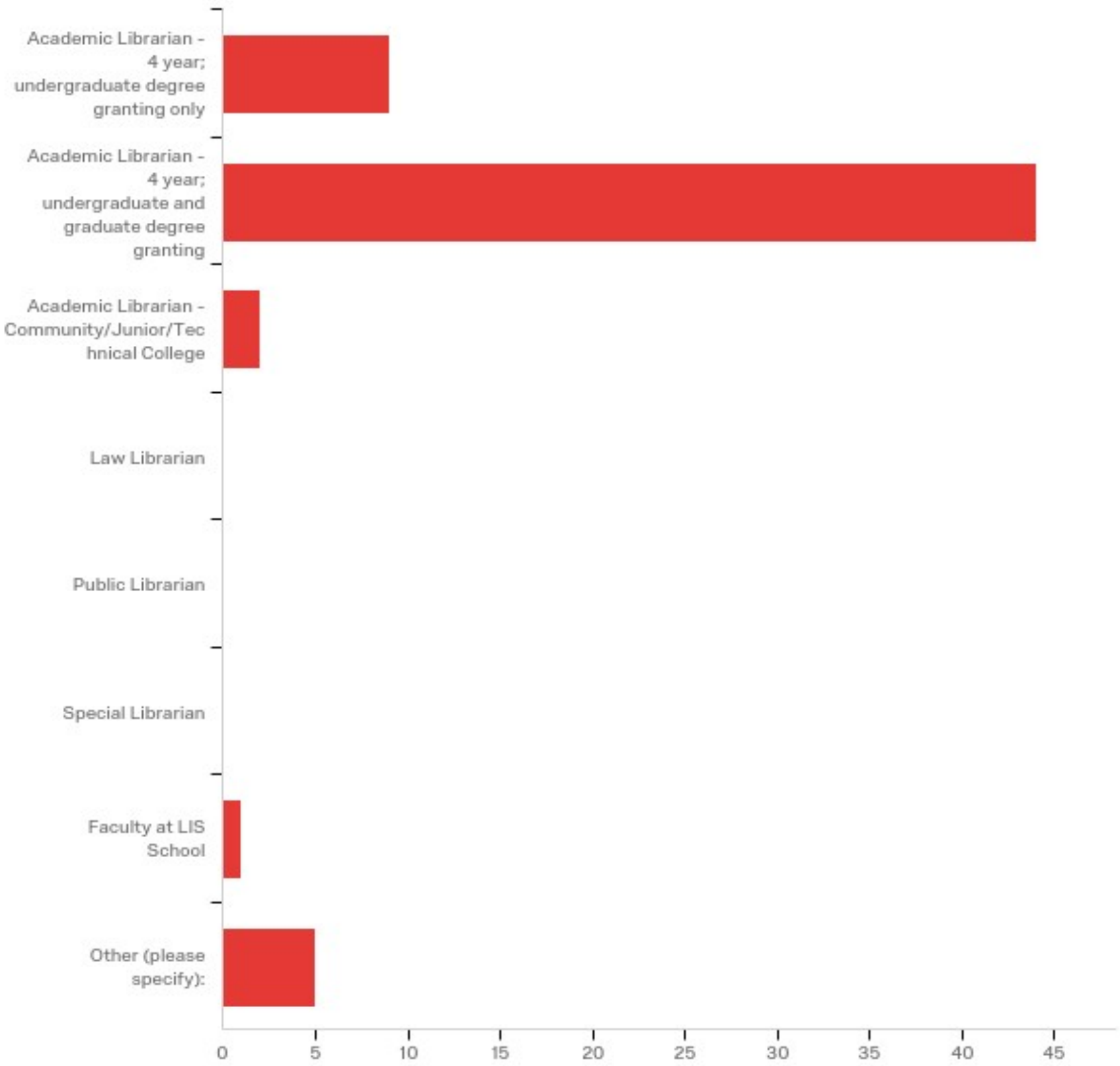

\begin{tabular}{r|r|r|r|}
\hline$\#$ & Answer & $\%$ & Count \\
\hline 1 & Academic Librarian - 4 year; undergraduate degree granting only & $14.75 \%$ & 9 \\
\hline 2 & Academic Librarian - 4 year; undergraduate and graduate degree granting & $72.13 \%$ & 44 \\
\hline 3 & Academic Librarian - Community/Junior/Technical College & $3.28 \%$ & 2 \\
\hline 4 & Law Librarian & $0.00 \%$ & 0 \\
\hline 5 & Public Librarian & $0.00 \%$ & 0 \\
\hline 6 & Special Librarian & $0.00 \%$ & 0 \\
\hline
\end{tabular}




\begin{tabular}{|l|r|r|r|}
\hline 7 & Faculty at LIS School & $1.64 \%$ & 1 \\
\hline 8 & Other (please specify): & $8.20 \%$ & 5 \\
\hline & Total & $100 \%$ & 61 \\
\hline
\end{tabular}

Other (please specify):

Other (please specify):

Special Collections, 4-year university; undergraduate and graduate dgree granting

Vendor

Retired LIS faculty

Associate Director - 4 year; undergraduate and graduate degree granting

Librarian in private institution 
Q7 - Please select all that apply to your institution's affiliation

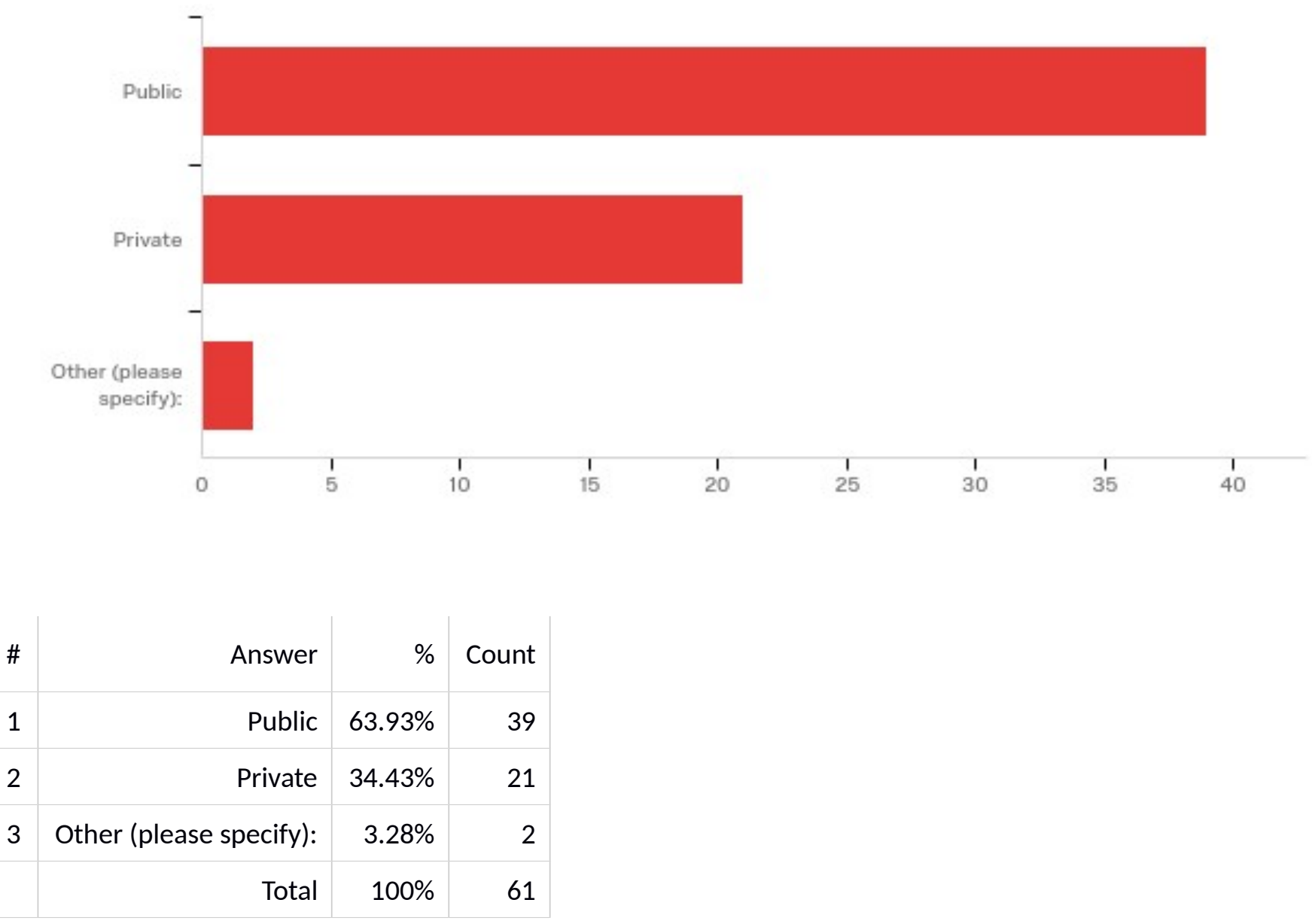

Other (please specify):

Other (please specify):

For-Profit

Land grant 
Q8 - How long have you been a librarian?

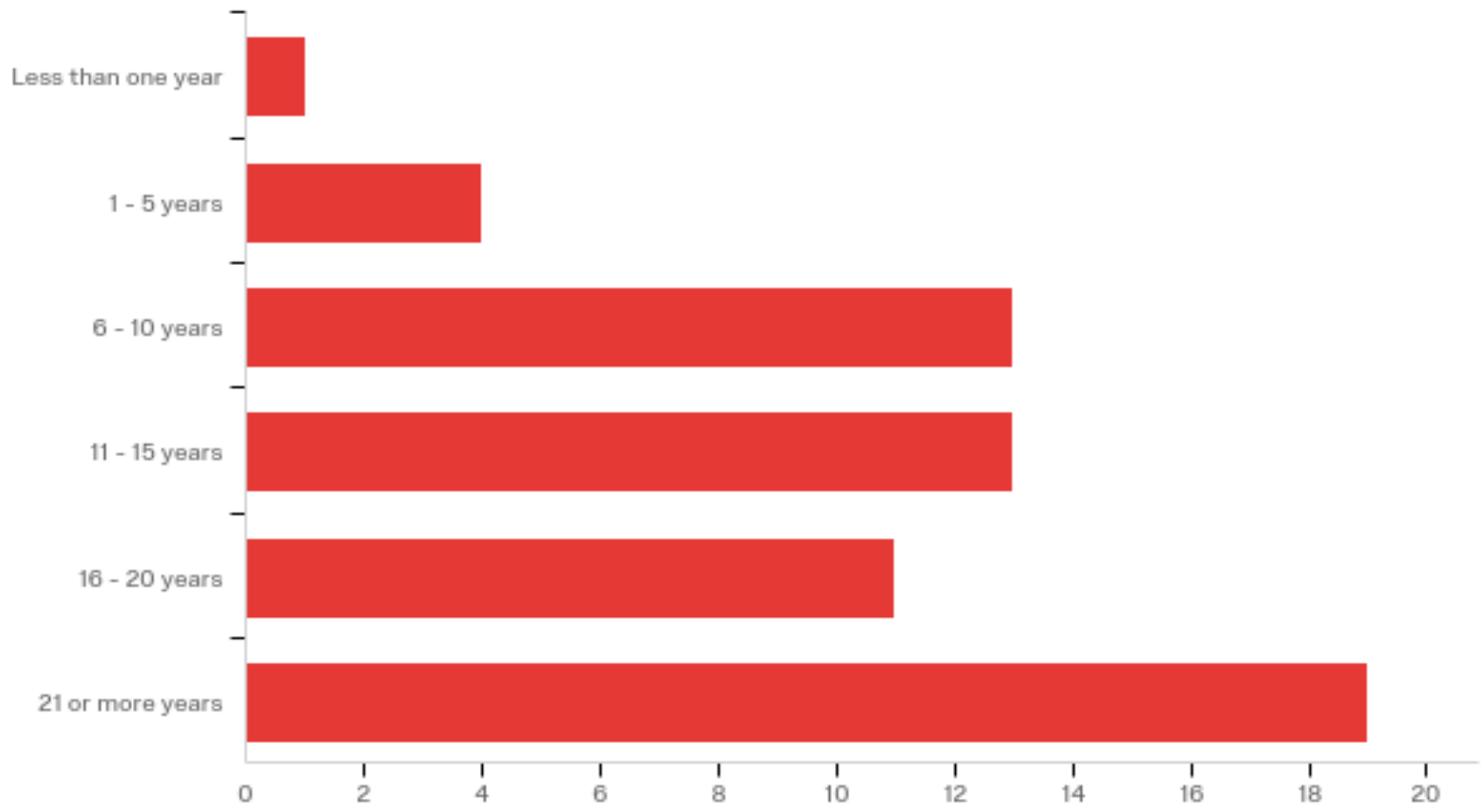

\begin{tabular}{r|r|r|r|}
\hline$\#$ & Answer & $\%$ & Count \\
\hline 1 & Less than one year & $1.64 \%$ & 1 \\
\hline 2 & $1-5$ years & $6.56 \%$ & 4 \\
\hline 3 & $6-10$ years & $21.31 \%$ & 13 \\
\hline 4 & $11-15$ years & $21.31 \%$ & 13 \\
\hline 5 & $16-20$ years & $18.03 \%$ & 11 \\
\hline 6 & 21 or more years & $31.15 \%$ & 19 \\
\hline & Total & $100 \%$ & 61 \\
\hline
\end{tabular}


Q10 - If you have faculty status, what is your rank?

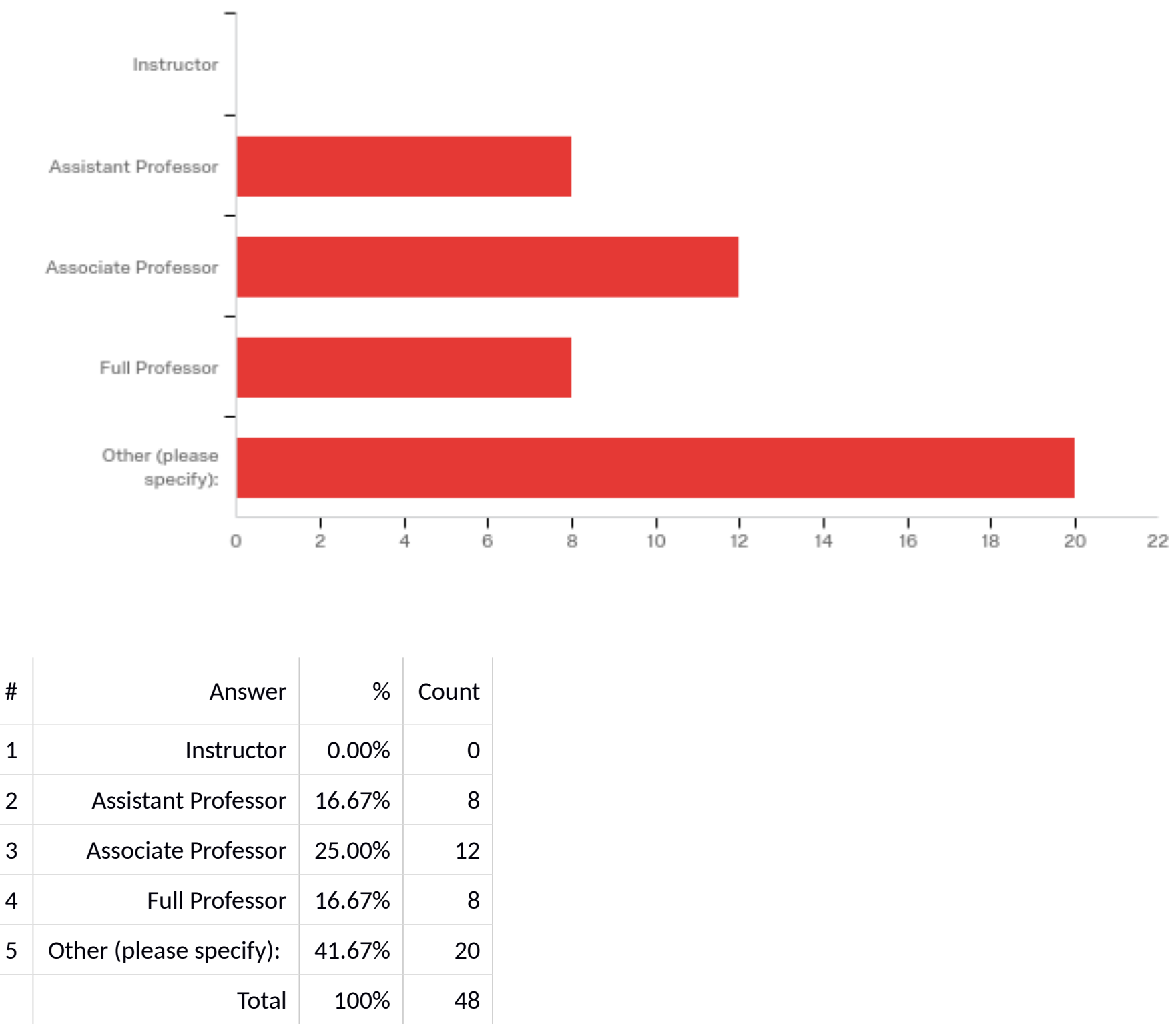

Other (please specify):

Other (please specify):

University Librarian

Staff

non faculty

Adjunct

Non Faculty

Professor Emeritus 


\section{Distinguished Professor / Librarian}

not applicable

Archivist

I do not have faculty status.

Non-ranked faculty

Retired LIS Full Professor

Assicuate Director

University Librarian

Associate Librarian -- not faculty status

Associate Librarian (faculty status)

Senior Assistant Librarian

Associate Librarian (we are faculty and the library has its own ranks and process) 
Q9 - What is your tenure status?

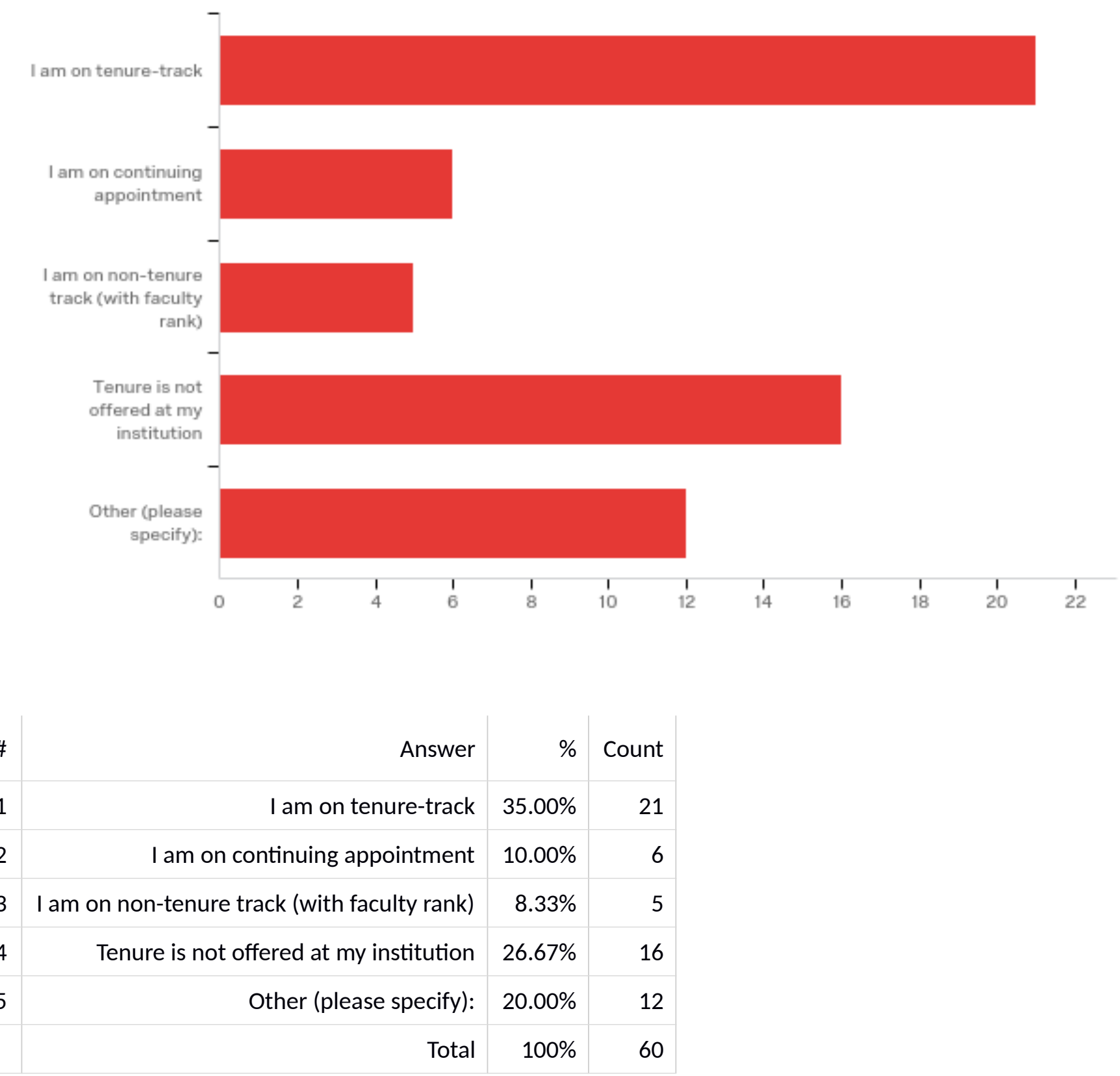

Other (please specify):

Other (please specify):

Senior Management Group/Administrator

Staff are not offered tenure at my institution

Retired (tenured)

Administrator, tenured 
tenured

I am tenured

I am an Academic Professional, which is essentially a non-tenure librarian

Not applicable

I do not have faculty status.

Some librarians are tenured (or on the tenure track). My position is not, it is considered Administration.

Retired 
Q11 - How many years total have you served on any editorial board?

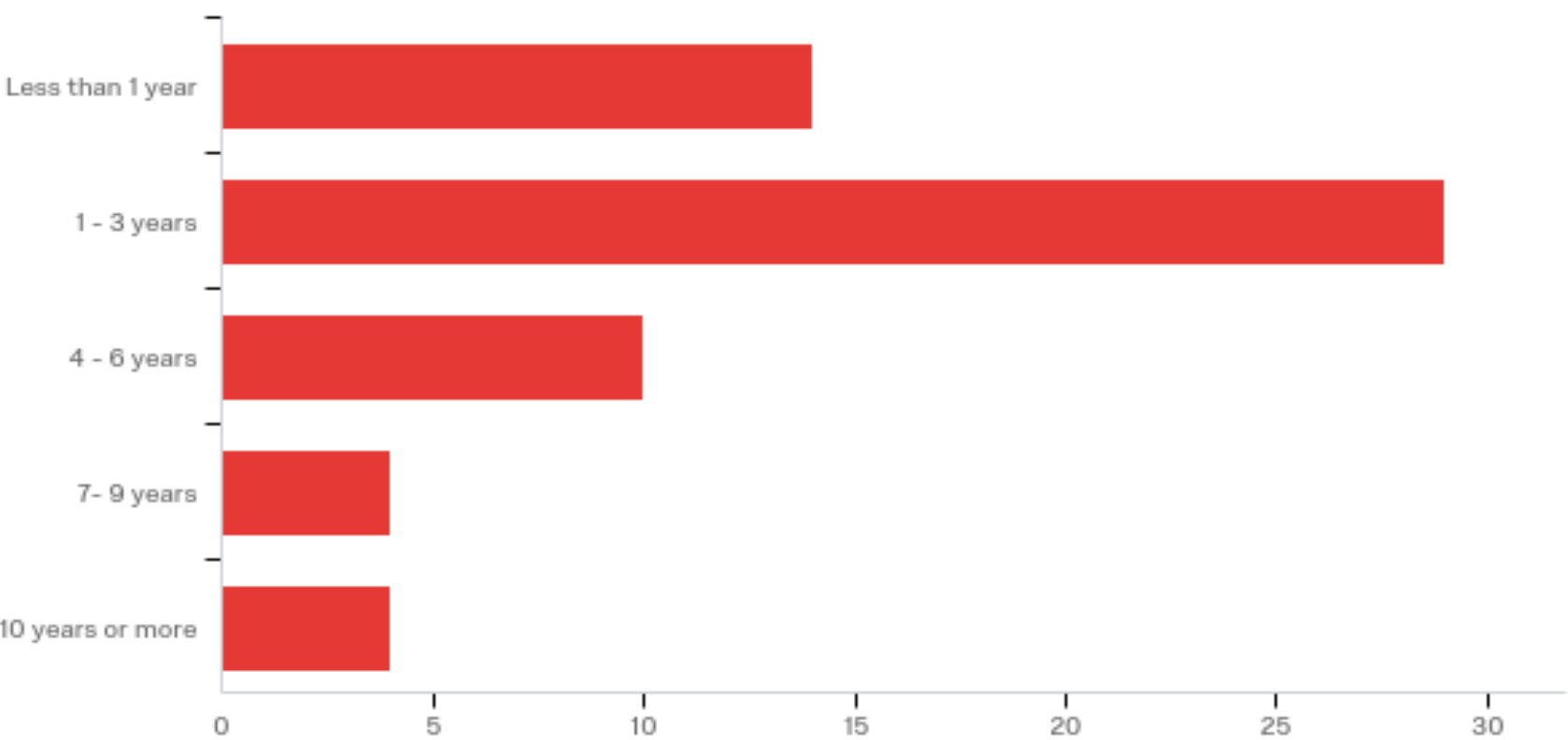

\begin{tabular}{|r|r|r|r|}
\hline$\#$ & Answer & $\%$ & Count \\
\hline 1 & Less than 1 year & $22.95 \%$ & 14 \\
\hline 2 & $1-3$ years & $47.54 \%$ & 29 \\
\hline 3 & $4-6$ years & $16.39 \%$ & 10 \\
\hline 4 & $7-9$ years & $6.56 \%$ & 4 \\
\hline 5 & 10 years or more & $6.56 \%$ & 4 \\
\hline & Total & $100 \%$ & 61 \\
\hline
\end{tabular}


Q6_8_TEXT - Topics

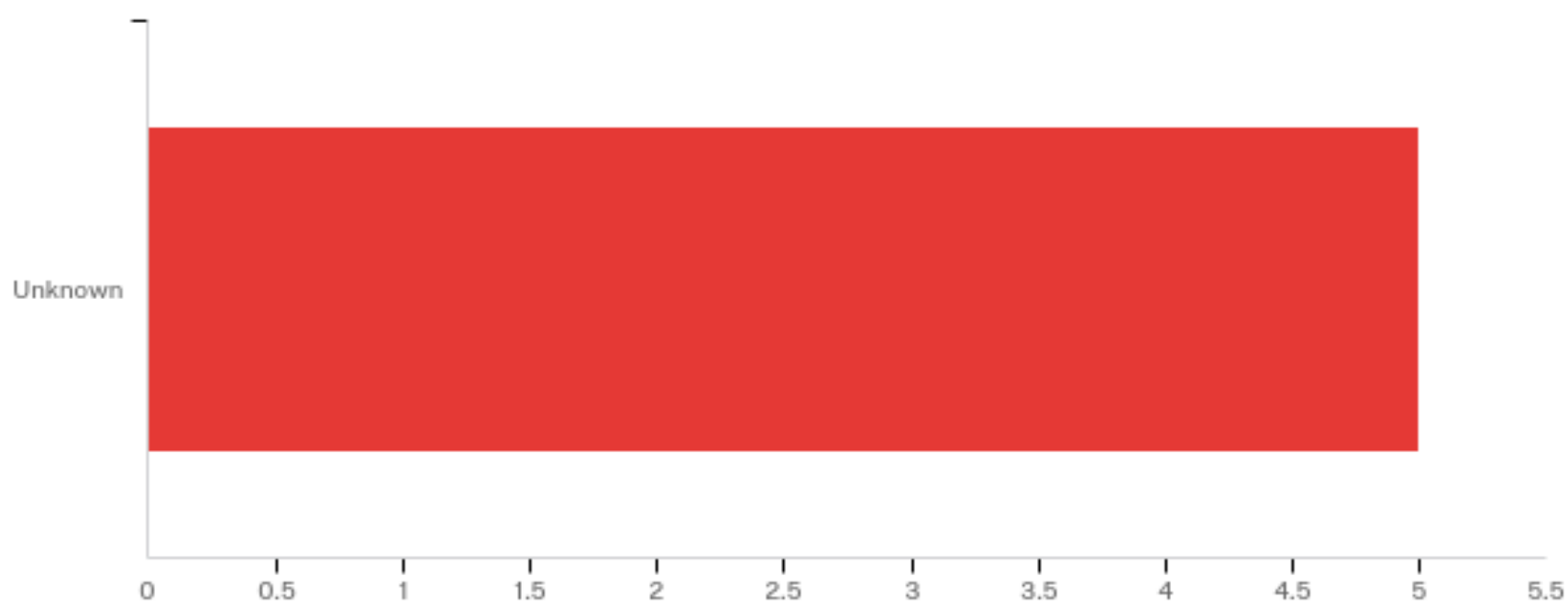

\begin{tabular}{l|r|r|} 
Answer & $\%$ & Count \\
\hline Unknown & $100.00 \%$ & 5 \\
\hline Total & $100 \%$ & 5 \\
\hline
\end{tabular}

